\title{
A Novel Serum Metabolomic Profile for the Differential Diagnosis of Distal Cholangiocarcinoma and Pancreatic Ductal Adenocarcinoma
}

\author{
Rocio I.R. Macias 1,2,*,+(D), Luis Muñoz-Bellvís ${ }^{3,+} \mathbb{D}^{\mathbb{D}}$, Anabel Sánchez-Martín ${ }^{1}$, Enara Arretxe ${ }^{4}$, \\ Ibon Martínez-Arranz ${ }^{4}{ }^{\mathbb{D}}$, Ainhoa Lapitz ${ }^{5}$, M. Laura Gutiérrez ${ }^{6}$, Adelaida La Casta ${ }^{5}$, \\ Cristina Alonso ${ }^{4}\left(\mathbb{D}\right.$, Luis M. González ${ }^{3}$, Matias A. Avila 2,7 (D, Maria L. Martinez-Chantar 2,8, \\ Rui E. Castro ${ }^{9} \mathbb{D}$, Luis Bujanda ${ }^{2,4}$, Jesus M. Banales ${ }^{2,5,10}$ and Jose J.G. Marin ${ }^{1,2} \mathbb{D}$
}

1 Experimental Hepatology and Drug Targeting (HEVEFARM) Group, University of Salamanca, Biomedical Research Institute of Salamanca (IBSAL), 37007 Salamanca, Spain; anabelsanchez@usal.es (A.S.-M.); jjgmarin@usal.es (J.J.G.M.)

2 National Institute for the Study of Liver and Gastrointestinal Diseases (CIBERehd, Carlos III Health Institute), 28029 Madrid, Spain; maavila@unav.es (M.A.A.); mlmartinez@cicbiogune.es (M.L.M.-C.); luis.bujandafernandezdepierola@osakidetza.eus (L.B.); jesus.banales@biodonostia.org (J.M.B.)

3 Service of General and Gastrointestinal Surgery, University Hospital of Salamanca, IBSAL, CIBERONC, 37007 Salamanca, Spain; luismb@usal.es (L.M.-B.); lgfcir@gmail.com (L.M.G.)

4 OWL Metabolomics, Bizkaia Technology Park, 48160 Derio, Spain; earretxe@owlmetabolomics.com (E.A.); imartinez@owlmetabolomics.com (I.M.-A.); calonso@owlmetabolomics.com (C.A.)

5 Department of Liver and Gastrointestinal Diseases, Biodonostia Health Research Institute, Donostia University Hospital, University of the Basque Country (UPV/EHU), 20014 San Sebastian, Spain; ainhoa.lapitz@biodonostia.org (A.L.); ADELAIDA.LACASTAMUNOA@osakidetza.eus (A.L.C.)

6 Department of Medicine and Cytometry Service (NUCLEUS), University of Salamanca, Cancer Research Center (IBMCC-CSIC/USAL), IBSAL, CIBERONC, 37007 Salamanca, Spain; mlgutierrez@usal.es

7 Program of Hepatology, Center for Applied Medical Research (CIMA), University of Navarra-IDISNA, 31008 Pamplona, Spain

8 Liver Disease Lab Center for Cooperative Research in Biosciences (CIC bioGUNE), Basque Research and Technology Alliance (BRTA), Bizkaia Technology Park, 48160 Derio, Spain

9 Research Institute for Medicines (iMed.ULisboa), Faculty of Pharmacy, Universidade de Lisboa, 1649-003 Lisbon, Portugal; ruieduardocastro@ff.ulisboa.pt

10 IKERBASQUE, Basque Foundation for Science, 48013 Bilbao, Spain

* Correspondence: rociorm@usal.es; Tel.: +34-666596621

+ These authors contributed equally to this work.

Received: 7 May 2020; Accepted: 29 May 2020; Published: 31 May 2020

\begin{abstract}
The diagnosis of adenocarcinomas located in the pancreas head, i.e., distal cholangiocarcinoma (dCCA) and pancreatic ductal adenocarcinoma (PDAC), constitutes a clinical challenge because they share many symptoms, are not easily distinguishable using imaging techniques and accurate biomarkers are not available. Searching for biomarkers with potential usefulness in the differential diagnosis of these tumors, we have determined serum metabolomic profiles in healthy controls and patients with dCCA, PDAC or benign pancreatic diseases (BPD). Ultra-high-performance liquid chromatography coupled to mass spectrometry (UHPLC-MS) analysis was performed in serum samples from dCCA $(n=34)$, PDAC $(n=38)$, BPD $(n=42)$ and control $(n=25)$ individuals, divided into discovery and validation cohorts. This approach permitted 484 metabolites to be determined, mainly lipids and amino acids. The analysis of the results led to the proposal of a logistic regression model able to discriminate patients with dCCA and PDAC (AUC value of 0.888) based on the combination of serum levels of nine metabolites (acylcarnitine $\mathrm{AC}(16: 0)$, ceramide Cer(d18:1/24:0), phosphatidylcholines PC(20:0/0:0) and PC(O-16:0/20:3), lysophosphatidylcholines PC(20:0/0:0) and PC(0:0/20:0), lysophosphatidylethanolamine PE(P-18:2/0:0), and sphingomyelins SM(d18:2/22:0) and $\mathrm{SM}(\mathrm{d} 18: 2 / 23: 0))$ and CA 19-9. In conclusion, we propose a novel specific panel of serum metabolites
\end{abstract}


that can help in the differential diagnosis of dCCA and PDAC. Further validation of their clinical usefulness in prospective studies is required.

Keywords: biliary cancer; differential diagnosis; metabolites; pancreatic cancer; tumor non-invasive biomarker

\section{Introduction}

Although distal cholangiocarcinoma (dCCA) and pancreatic ductal adenocarcinoma (PDAC) share a close anatomical location, they are considered distinct entities and require specific management strategies [1]. Whereas dCCA is an aggressive malignancy that arises in the biliary tract below the cystic duct and represents approximately $20 \%$ of CCAs, PDAC derives from the epithelium of pancreatic ducts and is the fourth cause of cancer-related deaths [2,3]. Although dCCA has a poor clinical outcome [4] due to its late diagnosis and resistance to chemotherapy [5], in general, the prognosis is worse in the case of PDAC [3].

Despite improvements in imaging techniques during recent years, the accurate diagnosis of adenocarcinomas located in the pancreas head area represents a clinical challenge in gastrointestinal oncology. Biopsy, either using cytologic brushing or fine-needle aspiration guided by endoscopic ultrasound, is mandatory to confirm the diagnosis. However, this has serious limitations: (i) repeat sampling is often required since the quality of the samples is not always sufficient to carry out the anatomopathological analysis, and (ii) the detection of malignant cells can confirm the diagnosis, but a negative result does not permit ruling it out [6]. To distinguish PDAC from benign pancreas diseases (BPD), such as chronic pancreatitis or pancreatic cysts, is also challenging, and the lack of accurate tumor biomarkers justifies that $\approx 5-10 \%$ of surgical removals of the head of the pancreas due to presumed malignancies are finally identified as benign lesions.

Several non-invasive biomarkers have been evaluated for the diagnosis of PDAC [7] and CCA [8,9], but none of them are being used in the clinical setting. Serum carbohydrate antigen 19-9 (CA 19-9) is the only FDA-approved biomarker for PDAC for both the follow-up of the therapeutic response [10] and for the detection of recurrence after surgery. Nevertheless, owing to its low sensitivity and specificity, CA 19-9 is far from being considered an optimal biomarker. Serum CA 19-9 is also used clinically to help in diagnosis and to monitor the response to therapy in biliary cancers, usually in combination with another unspecific marker, i.e., carcinoembryonic antigen (CEA). However, its accuracy is low and is not suitable for early detection. In addition, CA 19-9 can be elevated in patients with obstructive cholestasis, chronic liver and pancreatic diseases, and premalignant pancreatic lesions. Moreover, $\approx$ $10 \%$ of the Caucasian population with Lewis-negative phenotype do not express this biomarker [11].

Therefore, there is an urgent need to identify reliable minimally invasive biomarkers that can help in the differential diagnosis of dCCA and PDAC. An optimal biomarker would also be expected to contribute to the early detection of these cancers. The analysis of a large number of small metabolites in biological samples represents an interesting approach for identifying clinically relevant biomarkers for different diseases. In this context, the aim of the present study was to evaluate the usefulness of differences in serum metabolomic profiles between dCCA and PDAC, as well as between these severe malignancies and BPD and healthy individuals.

\section{Results}

\subsection{Characteristics of the Study Population}

The demographic and clinical features of individuals from both cohorts are shown in Table 1. The age was higher in patients with dCCA and PDAC than in patients with BPD and healthy individuals and only the latter group included a lower percentage of males. Most tumors included in the dCCA 
group were in early stage, while there was a similar distribution of tumors in early and advanced stage in the PDAC group. Regarding liver biochemical parameters (Table 1), a significant increase in ALT, GGT, alkaline phosphatase and total bilirubin was found in patients with dCCA and PDAC. Except for total bilirubin, these parameters were also found to be elevated in BPD, although the magnitude of changes was lower than that observed in patients with tumors.

Table 1. Demographic and clinical characteristics of the discovery and validation cohorts.

\begin{tabular}{|c|c|c|c|c|c|c|c|c|}
\hline \multirow[b]{2}{*}{ Variable } & \multicolumn{2}{|c|}{ Control } & \multicolumn{2}{|c|}{ BPD } & \multicolumn{2}{|c|}{ dCCA } & \multicolumn{2}{|c|}{ PDAC } \\
\hline & $\begin{array}{l}\text { Discovery } \\
(n=12)\end{array}$ & $\begin{array}{c}\text { Validation } \\
(n=13)\end{array}$ & $\begin{array}{c}\text { Discovery } \\
(n=22)\end{array}$ & $\begin{array}{l}\text { Validation } \\
(n=20)\end{array}$ & $\begin{array}{c}\text { Discovery } \\
(n=16)\end{array}$ & $\begin{array}{l}\text { Validation } \\
(n=18)\end{array}$ & $\begin{array}{c}\text { Discovery } \\
(n=19)\end{array}$ & $\begin{array}{l}\text { Validation } \\
(n=19)\end{array}$ \\
\hline $\begin{array}{l}\text { Age, } \\
\text { mean } \pm \\
\text { SD }\end{array}$ & $53.7 \pm 9.8$ & $52.2 \pm 8.6$ & $\begin{array}{c}60.9 \pm \\
12.3\end{array}$ & $\begin{array}{c}59.9 \pm \\
11.6\end{array}$ & $70.9 \pm 7.0$ & $\begin{array}{c}68.6 \pm \\
10.9\end{array}$ & $68.5 \pm 9.3$ & $63.1 \pm 9.4$ \\
\hline $\begin{array}{l}\text { Males, } n \\
(\%)\end{array}$ & $5(41.7)$ & $6(46.1)$ & $12(54.5)$ & $10(50)$ & $12(75)$ & $10(55.6)$ & $12(63.1)$ & $12(63.1)$ \\
\hline \multicolumn{9}{|c|}{ Tumor stage ${ }^{*}, n(\%)$} \\
\hline I & - & - & - & - & $0(0)$ & $1(5.6)$ & $1(5.3)$ & $2(10.5)$ \\
\hline II & - & - & - & - & $13(81.2)$ & $16(88.8)$ & $7(36.8)$ & 7 (36.8) \\
\hline III & - & - & - & - & $1(6.3)$ & $0(0)$ & $2(10.5)$ & $5(26.3)$ \\
\hline IV & - & - & - & - & $2(12.5)$ & $1(5.6)$ & $9(47.4)$ & $5(26.3)$ \\
\hline \multicolumn{9}{|c|}{ Biochemistry, mean \pm SD } \\
\hline $\begin{array}{c}\text { ALT } \\
(\mathrm{IU} / \mathrm{L})\end{array}$ & $21.3 \pm 8.0$ & $17.1 \pm 6.7$ & $\begin{array}{c}38.9 \pm \\
64.3\end{array}$ & $\begin{array}{c}32.0 \pm \\
27.0\end{array}$ & $107 \pm \underset{a, b}{ } 118$ & $\begin{array}{c}38.5 \pm \\
94.5\end{array}$ & $207 \pm 203$ & $\begin{array}{c}208 \pm 318 \\
\mathrm{a}, \mathrm{b}\end{array}$ \\
\hline $\begin{array}{l}\text { GGT } \\
\text { (IU/L) }\end{array}$ & $\begin{array}{c}24.8 \pm \\
20.4\end{array}$ & $19.0 \pm 9.8$ & $\begin{array}{c}125 \pm 206 \\
a\end{array}$ & $\begin{array}{c}100 \pm 142 \\
\mathrm{a}\end{array}$ & $\begin{array}{c}589 \pm 592 \\
\mathrm{a}, \mathrm{b}\end{array}$ & $\underset{\mathrm{a}, \mathrm{b}}{411 \pm 816}$ & $\begin{array}{c}775 \pm \\
1037^{a, b}\end{array}$ & $\begin{array}{c}784 \pm 978 \\
\mathrm{a}, \mathrm{b}\end{array}$ \\
\hline $\begin{array}{l}\text { Alkaline } \\
\text { phosphatase } \\
\text { (IU/L) }\end{array}$ & $\begin{array}{c}56.1 .8 \pm \\
15.4\end{array}$ & $\begin{array}{c}58.5 \pm \\
22.9\end{array}$ & $102 \pm 115$ & $94 \pm 68$ & $\underset{\mathrm{a}, \mathrm{b}}{301 \pm 220}$ & $\begin{array}{c}207 \pm 153 \\
a\end{array}$ & $\underset{\mathrm{a}, \mathrm{b}}{442 \pm 427}$ & $\begin{array}{c}380 \pm 359 \\
\mathrm{a}, \mathrm{b}\end{array}$ \\
\hline $\begin{array}{c}\text { Total } \\
\text { bilirubin } \\
(\mathrm{mg} / \mathrm{dL})\end{array}$ & $0.5 \pm 0.3$ & $0.6 \pm 0.2$ & $0.5 \pm 0.2$ & $0.8 \pm 1.7$ & $\underset{\mathrm{a}, \mathrm{b}}{6.9 \pm 7.5}$ & $2.8 \pm 4.9$ & $\underset{\mathrm{a}, \mathrm{b}}{7.0 \pm 7.6}$ & $\underset{\mathrm{a}, \mathrm{b}}{7.3 \pm 6.2}$ \\
\hline $\begin{array}{l}\text { CA 19-9 } \\
(\mathrm{IU} / \mathrm{mL})\end{array}$ & $5.4 \pm 4.5$ & $8.6 \pm 7.4$ & $\begin{array}{l}46.6 \pm \\
74.9^{a}\end{array}$ & $\begin{array}{c}15.1 \pm \\
10.5\end{array}$ & $\begin{array}{c}893 \pm \\
2405^{a, b}\end{array}$ & $\begin{array}{c}328 \pm 855 \\
\mathrm{a}, \mathrm{b}\end{array}$ & $\begin{array}{c}2983 \pm \\
8024^{a, b}\end{array}$ & $\underset{\mathrm{a}, \mathrm{b}}{431 \pm 561}$ \\
\hline
\end{tabular}

$\mathrm{a}, p<0.05$ compared with control (in the same cohort) and ${ }^{\mathrm{b}}, p<0.05$ compared with BPD (in the same cohort) using the Bonferroni method of multiple range test. *, AJCC Cancer Staging Manual, 7th Edition. ALT; alanine aminotransferase, BPD, benign pancreatic disease; CA 19-9, carbohydrate antigen 19-9; dCCA, distal cholangiocarcinoma; GGT, gamma-glutamyl transpeptidase; PDAC, pancreatic ductal adenocarcinoma.

A significant increase in serum levels of CA 19-9 was found in both dCCA and PDAC, with a marked interindividual variability. Moreover, although CA 19-9 levels were also elevated in some patients with BPD, both with pancreatic cysts and with chronic pancreatitis, these were significantly lower than those found in patients with cancer.

Any clustering of the different groups of samples according to the serum metabolome was evaluated using multivariate data analysis, unsupervised principal component analysis (PCA) and supervised orthogonal partial least-squares to latent structures discriminant analysis (OPLS-DA) approaches. As shown in Figure 1, no differences in serum metabolomic profiles were found between the hospitals of origin, discovery and validation cohorts, gender, and group of age or group of samples (Figure 1A-E, respectively). A random distribution of patients with cysts and pancreatitis, both included in the BPD group, was found (Figure 1F). 

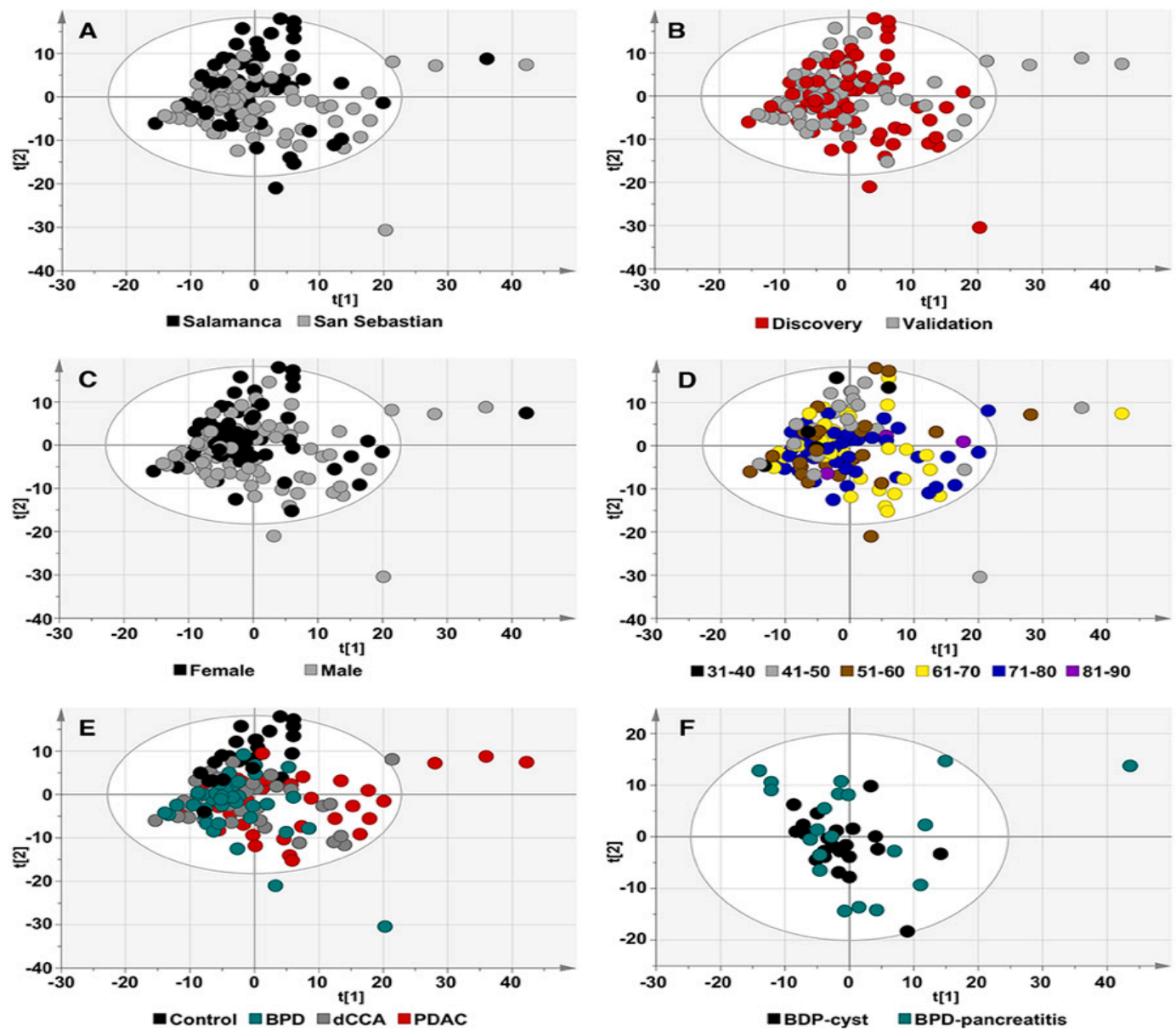

Figure 1. Principal component analysis (PCA) score plots of human serum samples. Colors represent (A) the origin of the samples, (B) discovery or validation cohort, (C) gender, (D) age range, (E) group of samples and (F) type of benign pancreatic disease (BPD). (A-E) Principal component 1 (t[1]) and principal component $2(\mathrm{t}[2])$ explain $17.4 \%$ and $11.2 \%$ of the total variance, respectively. $(\mathbf{F}) \mathrm{t}[1]$ and $\mathrm{t}[2]$ explain $19.8 \%$ and $13.1 \%$ of the total variance, respectively. Each dot represents one sample. The ellipse represents $95 \%$ confidence interval according to Hotelling's $\mathrm{T}^{2}$ test.

The supervised OPLS-DA model showed a good predictive ability to discriminate patient groups from healthy individuals, since $\mathrm{Q}^{2} \mathrm{X}=0.694$ (Figure 2A), triglycerides and, to a lesser extent, oxidized fatty acids and bile acids (all of them increased) and sphingomyelins and glycerophosphatidylcholines (both decreased) being the main contributors to the differences found between patients and control individuals. However, the supervised OPLS-DA models to differentiate dCCA vs. BPD patients, PDAC vs. BPD and both types of tumors showed very low predictive ability (Figure 2B-D, respectively), since $\mathrm{Q}^{2} \mathrm{X}$ values were low, especially in the comparisons of PDAC with $\mathrm{BPD}\left(\mathrm{Q}^{2} \mathrm{X}=0.163\right)$ and dCCA $\left(\mathrm{Q}^{2} \mathrm{X}\right.$ close to 0$)$. 

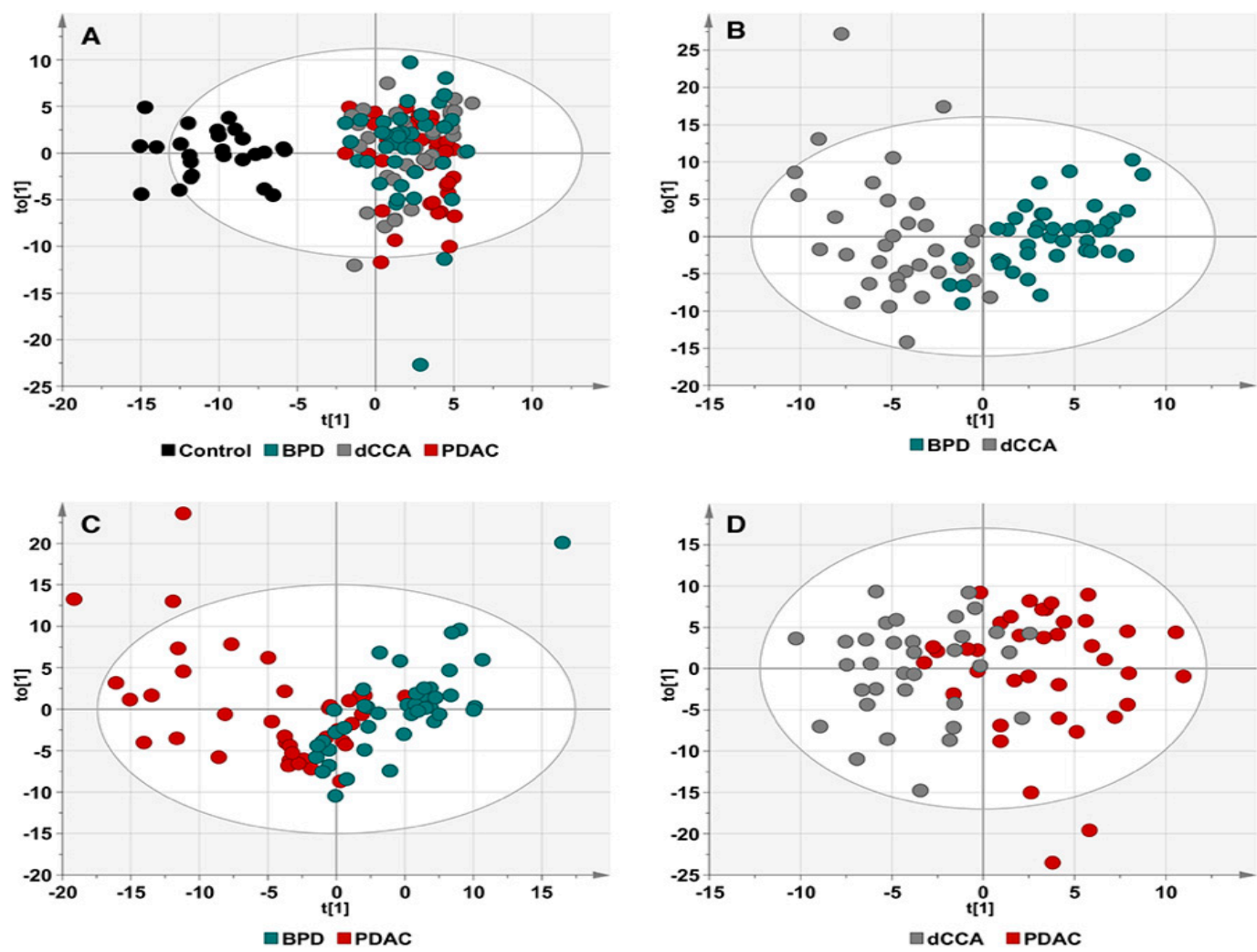

Figure 2. Score plots for the first predictive (t[1]) and orthogonal (to[1]) components of the supervised orthogonal partial least squares discriminant analysis (OPLS-DA) models for (A) disease vs. control samples; $\left.R^{2} X=0.445 ; R^{2} Y=0.835 ; Q^{2} X=0.694\right)$, (B) dCCA vs. BPD samples; $R^{2} X=0.315 ; R^{2} Y=0.697$; $\mathrm{Q}^{2} \mathrm{X}=0.425$, (C) PDAC vs. BPD samples; $\mathrm{R}^{2} \mathrm{X}=0.265 ; \mathrm{R}^{2} \mathrm{Y}=0.471 ; \mathrm{Q}^{2} \mathrm{X}=0.163$, and (D) dCCA vs. PDAC samples; $R^{2} X=0.24 ; R^{2} Y=0.527 ; Q^{2} X=0.036$. Each dot represents one sample. The ellipse represents $95 \%$ confidence interval according to Hotelling's $\mathrm{T}^{2}$ test.

\subsection{Serum Metabolomic Profiles of Patients with $d C C A, P D A C$ and BPD and Healthy Individuals}

During the discovery phase we were able to determine 484 metabolites in serum samples, which was confirmed in the validation cohort. Changes in the levels of molecules belonging to the different families of analyzed metabolites (lipids, amino acids and amino acids derivatives) were found. Figure 3 depicts the heatmaps showing the fold-changes and the $p$-values generated from different two-groups comparisons carried out in the discovery and validation cohorts, and considering all samples together. 


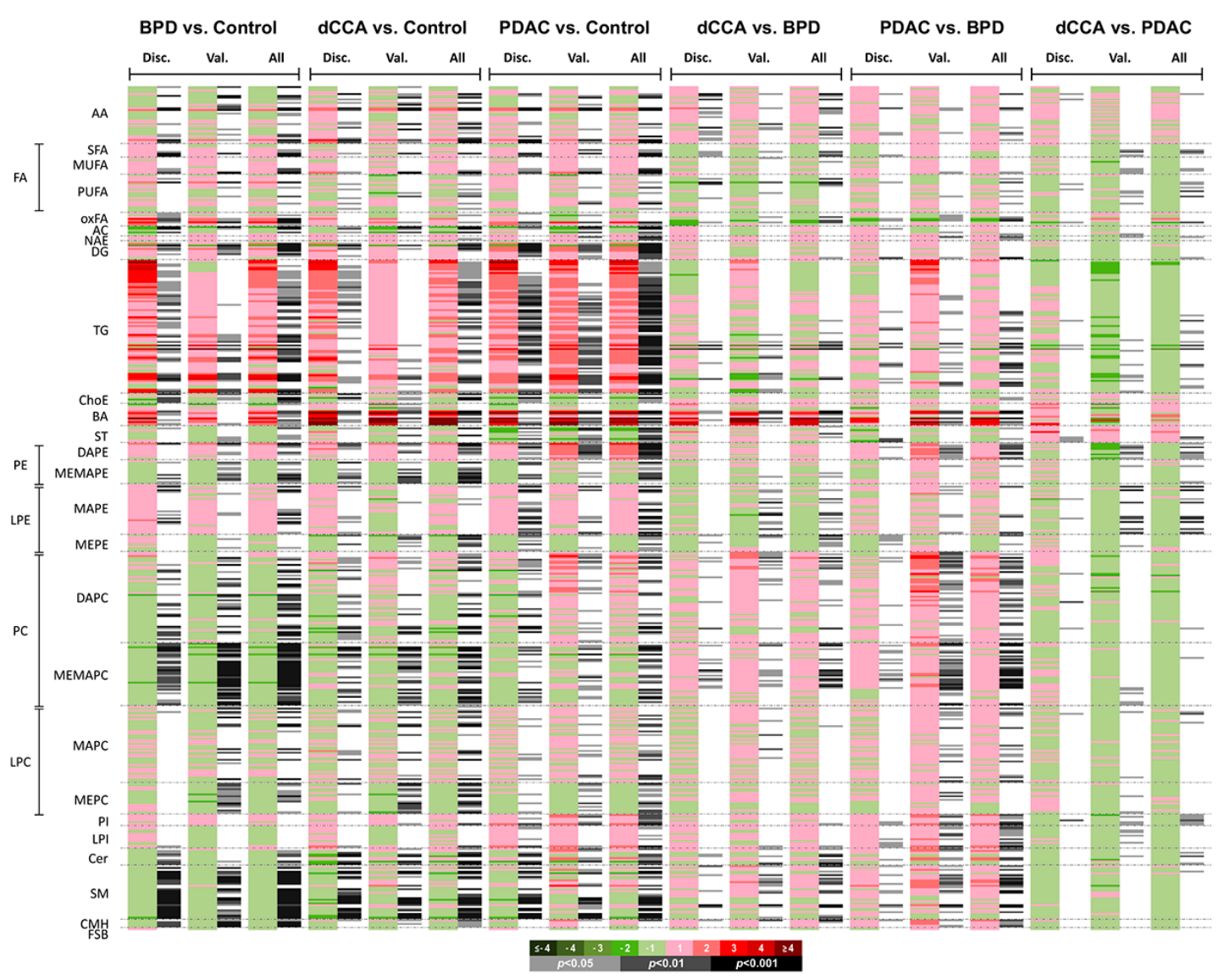

Figure 3. Metabolomic signatures in serum of patients with dCCA, PDAC or BPD and healthy individuals (Control). Heatmaps show fold-changes and $p$-values in each two-group comparison of the relative metabolite levels in serum samples in the discovery cohort (Disc.), in the validation cohort (Val.) and considering all samples together (All). The $\log _{2}$ transformed metabolite abundance ratios are depicted for each comparison. In the scale, colors from green to red correspond to drop or elevation of metabolite levels and gray lines show significant fold-changes of individual metabolites; darker gray colors indicate higher significance. Metabolites are grouped by chemical group/subgroup: AA, amino acids; AC, acylcarnitines; BA, bile acids; Cer, ceramides; ChoE, cholesteryl esters; $\mathrm{CMH}$, monohexosylceramides; DAPC, diacylglycerophosphocholines; DAPE, diacylglycerophosphoethanolamines; DG, diglycerides; FSB, free sphingoid bases; LPC, lysophosphatidylcholines; LPE, lysophosphatidylethanolamines; LPI, lysophosphatidylinositols MAPC, monoacylglycerophosphatidylcholines; MAPE, monoacylglycerophosphatidylethanolamines; MEMAPC, 1-ether, 2-acylglycerophosphatidylcholines; MEMAPE, 1-ether, 2-acylglycerophospha tidylethanolamines; MEPC; 1- monoetherglycerophosphatidylcholines; MEPE, 1-monoetherglycer ophosphatidylethanolamines; MUFA, monounsaturated fatty acids; NAE, N-acyl ethanolamines; oxFA, oxidized fatty acids; PC, phosphatidylcholines; PE, phosphatidylethanolamines; PI, phosphatidylinositols; PUFA, polyunsaturated fatty acids; SFA, saturated fatty acids; SM, sphingomyelins; ST, steroids; TG, triglycerides.

Figure 4 shows the volcano plots generated for each two-groups comparison, and the number of metabolites significantly changed in each comparison considering the full cohort (Figure 4G). When BDL was compared with control, altered serum concentrations of 268 metabolites (mainly phosphatidylcholines $>$ triglycerides $>$ sphingomyelins $\approx$ lysophosphatidylcholines) were found. The comparison of dCCA with control revealed altered serum levels of 236 metabolites (mainly triglycerides $\approx$ phosphatidylcholines $>$ lysophosphatidylcholines $>$ sphingomyelins). 

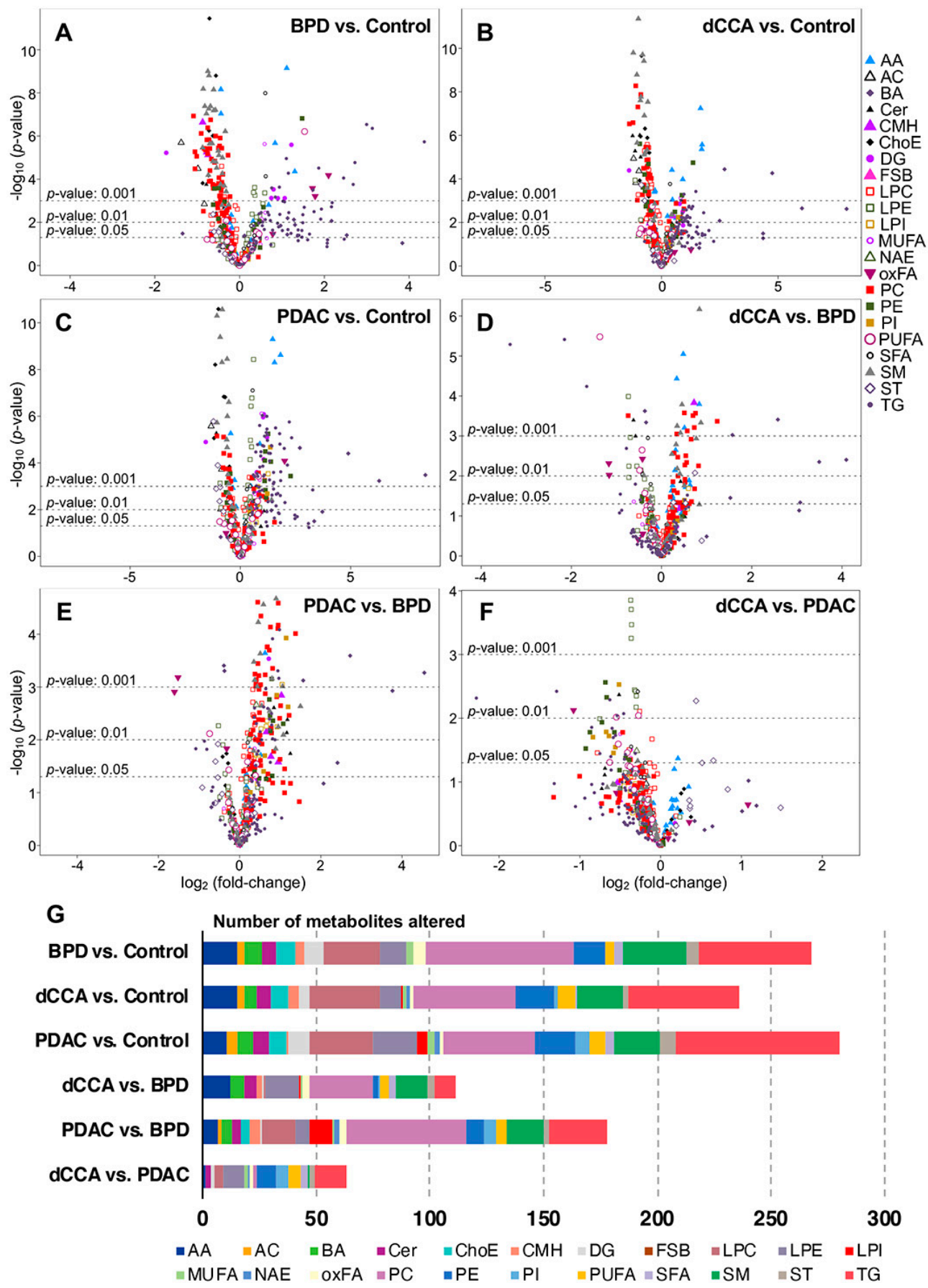

Figure 4. Volcano plots $\left[-\log _{10}\left(p\right.\right.$-value) and $\log _{2}$ (fold-change)] considering the serum metabolite levels of the whole cohort of (A) BPD patients vs. controls, (B) dCCA vs. controls, (C) PDAC vs. controls, (D) dCCA vs. BPD, (E) PDAC vs. BPD and (F) dCCA vs. PDAC. (G) Number of metabolites and metabolite classes significantly different in each comparison. AA, amino acids; AC, acylcarnitines; BA, bile acids; Cer, ceramides; ChoE, cholesteryl esters; CMH, monohexosylceramides; DG, diglycerides; FSB, free sphingoid bases; LPC, lysophosphatidylcholines; LPE, lysophosphatidylethanolamines; LPI, lysophosphatidylinositols; MUFA, monounsaturated fatty acids; NAE, N-acyl ethanolamines; oxFA, oxidized fatty acids; PC, phosphatidylcholines; PE, phosphatidylethanolamines; PI, phosphatidylinositols; PUFA, polyunsaturated fatty acids; SFA, saturated fatty acids; SM, sphingomyelins; ST, steroids; TG, triglycerides.

The highest number of metabolites affected by changes in their serum levels ( $n=280$; mainly triglycerides $>$ phosphatidylcholines $>$ lysophosphatidylcholines) was found in the PDAC group. 
Different serum levels of 111 metabolites were found when comparing dCCA with BPD (mainly phosphatidylcholines $>$ lysophosphatidyletanolamines = sphingomyelins), whereas this number increased to 178 when comparing PDAC with BPD (mainly phosphatidylcholines $>$ triglycerides). The number of serum metabolites altered when comparing dCCA vs. PDAC was 63 (mainly triglycerides $>$ phosphatidyletanolamines > lysophosphatidyletanolamines), and most of them were higher in PDCA than in dCCA. The number of metabolites with a value of area under the receiver operating characteristic curve (AUC) $\geq 0.8$ was 73 when comparing BPD vs. control, 63 when comparing dCCA vs. control and 72 when comparing PDAC vs. control.

An important number of metabolites were found altered in the serum of more than one group of patients, although the magnitude of changes was higher in patients with cancer. Table 2 shows the 10 metabolites with the best diagnostic capacity (best values of AUC, sensitivity and specificity) for each disease vs. control. Complete panels are presented in Table S1A-C.

Table 2. Diagnostic capacity of the top 10 metabolites in the comparison of each disease vs. control considering the whole cohort.

\begin{tabular}{|c|c|c|c|c|c|}
\hline \multirow{11}{*}{ 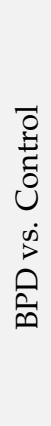 } & Metabolite & AUC & Sensitivity & Specificity & $\log _{2} \mathrm{FC}$ \\
\hline & Glutamic acid & 0.926 & 90 & 84 & 1.112 \\
\hline & Tryptophan & 0.910 & 92 & 83 & -0.441 \\
\hline & DG(34:0) & 0.910 & 84 & 86 & -1.731 \\
\hline & PE(16:0/18:1) & 0.909 & 88 & 88 & 1.475 \\
\hline & $\operatorname{SM}(32: 1)$ & 0.909 & 96 & 74 & -0.722 \\
\hline & $\mathrm{AC}(8: 0)$ & 0.906 & 92 & 86 & -1.379 \\
\hline & $\mathrm{PC}(\mathrm{O}-16: 0 / 18: 2)$ & 0.903 & 92 & 71 & -1.087 \\
\hline & Arachidic acid & 0.898 & 69 & 96 & 0.606 \\
\hline & $\mathrm{SM}(\mathrm{d} 18: 2 / 22: 0)$ & 0.896 & 84 & 83 & -0.749 \\
\hline & $\operatorname{SM}(38: 1)$ & 0.889 & 96 & 76 & -0.612 \\
\hline \multirow{11}{*}{ 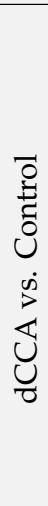 } & Metabolite & AUC & Sensitivity & Specificity & $\log _{2} \mathrm{FC}$ \\
\hline & $\mathrm{SM}(\mathrm{d} 18: 2 / 22: 0)$ & 0.967 & 92 & 94 & -0.992 \\
\hline & $\mathrm{SM}(\mathrm{d} 18: 2 / 23: 0)$ & 0.959 & 88 & 97 & -1.204 \\
\hline & $\operatorname{SM}(39: 1)$ & 0.958 & 96 & 91 & -1.052 \\
\hline & Aspartic acid & 0.955 & 79 & 100 & 1.671 \\
\hline & $\begin{array}{c}\text { Glycocholic } \\
\text { acid }\end{array}$ & 0.954 & 94 & 88 & 4.779 \\
\hline & $\operatorname{SM}(38: 1)$ & 0.951 & 96 & 94 & -0.750 \\
\hline & $\mathrm{SM}(\mathrm{d} 18: 1 / 23: 0)$ & 0.929 & 80 & 94 & -0.900 \\
\hline & $\mathrm{SM}(\mathrm{d} 18: 1 / 22: 0)$ & 0.928 & 92 & 88 & -0.810 \\
\hline & $\mathrm{SM}(\mathrm{d} 18: 2 / 20: 0)$ & 0.921 & 84 & 88 & -0.581 \\
\hline & $\begin{array}{l}\text { Taurocholic } \\
\text { acid }\end{array}$ & 0.919 & 76 & 100 & 8.035 \\
\hline \multirow{11}{*}{ 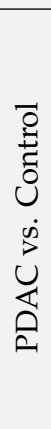 } & Metabolite & AUC & Sensitivity & Specificity & $\log _{2} \mathrm{FC}$ \\
\hline & Glutamic acid & 0.937 & 92 & 88 & 1.570 \\
\hline & Aspartic acid & 0.937 & 79 & 96 & 1.473 \\
\hline & PE(16:0/18:1) & 0.919 & 82 & 88 & 2.295 \\
\hline & $\mathrm{SM}(\mathrm{d} 18: 2 / 22: 0)$ & 0.919 & 92 & 89 & -0.810 \\
\hline & $\operatorname{SM}(39: 1)$ & 0.915 & 88 & 82 & -0.907 \\
\hline & $\mathrm{SM}(\mathrm{d} 18: 2 / 23: 0)$ & 0.911 & 92 & 87 & -1.059 \\
\hline & ChoE(18:3) & 0.907 & 76 & 92 & -1.178 \\
\hline & $\mathrm{AC}(8: 0)$ & 0.903 & 80 & 92 & -1.312 \\
\hline & $\operatorname{SM}(38: 1)$ & 0.899 & 96 & 76 & -0.603 \\
\hline & $\operatorname{PE}(16: 0 / 0: 0)$ & 0.897 & 79 & 92 & 0.601 \\
\hline
\end{tabular}

AUC, area under the receiver operating characteristic curve; FC, fold change. Colors from green to red correspond to drop or elevation of metabolite levels.

Although fewer alterations in the circulating metabolomic profiles were observed when the different diseases were cross compared, we found changes with interest in diagnosis. Among 50 metabolites with significant AUC values in the comparison of dCCA vs. BPD, 6 showed AUC values 
of $\geq 0.8$ (Table 3), while 2 among 61 in the comparison PDAC vs. BPD reached these AUC values. In the comparison dCCA vs. PDAC, 9 metabolites showed significant AUC values, although all with AUC $<0.8$. In the last comparison serum concentrations of the 9 metabolites were lower in dCCA than in PDAC. Table 3 shows the 9-10 metabolites with the best diagnostic capacity in each two-groups comparison, and the complete panels are presented in Table S1D,E.

Table 3. Diagnostic capacity of the top 9-10 metabolites in each two-disease group comparison considering the whole cohort.

\begin{tabular}{|c|c|c|c|c|c|}
\hline \multirow{11}{*}{$\begin{array}{l}\hat{\infty} \\
\dot{\infty} \\
\dot{\vec{j}} \\
\dot{U} \\
\dot{0}\end{array}$} & Metabolite & AUC & Sensitivity & Specificity & $\log _{2} \mathrm{FC}$ \\
\hline & SM(d18:1/23:1) & 0.858 & 79 & 81 & 0.839 \\
\hline & $\begin{array}{c}\text { Glycocholic } \\
\text { acid }\end{array}$ & 0.834 & 94 & 62 & 2.579 \\
\hline & Taurocholic & 0833 & 73 & 83 & 4096 \\
\hline & $\operatorname{PC}(16: 0 / 16: 0)$ & 0.811 & 76 & 79 & 0.753 \\
\hline & $P C(31: 0)$ & 0.805 & 71 & 81 & 1.233 \\
\hline & TG(54:7) & 0.800 & 60 & 91 & -3.358 \\
\hline & $18: 3 n-3$ & 0.790 & 69 & 82 & -1.368 \\
\hline & $\mathrm{CMH}(\mathrm{d} 18: 1 / 16: 0)$ & 0.788 & 82 & 71 & 0.720 \\
\hline & Phenylalanine & 0.785 & 56 & 90 & 0.482 \\
\hline & TG(54:6) & 0.783 & 51 & 100 & -2.154 \\
\hline \multirow{11}{*}{ 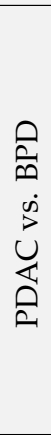 } & Metabolite & AUC & Sensitivity & Specificity & $\log _{2} \mathrm{FC}$ \\
\hline & $\mathrm{PC}(\mathrm{O}-34: 1)$ & 0.814 & 66 & 90 & 0.951 \\
\hline & $\mathrm{SM}(\mathrm{d} 18: 1 / 23: 1)$ & 0.813 & 79 & 79 & 0.898 \\
\hline & PC(P-16:0/16:0) & 0.795 & 74 & 74 & 0.730 \\
\hline & PC $(16: 0 / 16: 0)$ & 0.794 & 66 & 86 & 0.950 \\
\hline & $\mathrm{PC}(31: 0)$ & 0.794 & 68 & 81 & 1.376 \\
\hline & PC $(\mathrm{O}-16: 0 / 16: 0)$ & 0.782 & 68 & 81 & 0.941 \\
\hline & $\mathrm{PC}(\mathrm{O}-38: 5)$ & 0.782 & 58 & 93 & 0.446 \\
\hline & PC $(\mathrm{O}-18: 1 / 18: 1)$ & 0.776 & 63 & 81 & 0.760 \\
\hline & PC $(\mathrm{O}-22: 1 / 20: 4)$ & 0.768 & 74 & 71 & 0.677 \\
\hline & $\mathrm{SM}(\mathrm{d} 18: 0 / 15: 0)$ & 0.766 & 55 & 93 & 1.058 \\
\hline \multirow{10}{*}{ 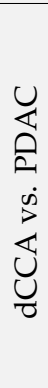 } & Metabolite & AUC & Sensitivity & Specificity & $\log _{2} \mathrm{FC}$ \\
\hline & $\mathrm{PE}(18: 0 / 0: 0)$ & 0.769 & 82 & 71 & -0.367 \\
\hline & $\operatorname{PE}(0: 0 / 18: 0)$ & 0.763 & 84 & 68 & -0.358 \\
\hline & $\operatorname{PE}(16: 0 / 0: 0)$ & 0.742 & 74 & 68 & -0.356 \\
\hline & $\operatorname{PE}(20: 4 / 0: 0)$ & 0.739 & 74 & 71 & -0.317 \\
\hline & $\operatorname{PE}(0: 0 / 20: 4)$ & 0.735 & 76 & 71 & -0.302 \\
\hline & $\operatorname{PE}(0: 0 / 16: 0)$ & 0.732 & 89 & 47 & -0.365 \\
\hline & $\operatorname{PE}(38: 5)$ & 0.719 & 95 & 44 & -0.844 \\
\hline & TG(52:5) & 0.705 & 71 & 68 & -0.620 \\
\hline & PE 20:4 & 0.704 & 95 & 38 & -0.520 \\
\hline
\end{tabular}

AUC, area under the receiver operating characteristic curve; FC, fold change. Colors from green to red correspond to drop or elevation of metabolite levels.

\subsection{Discrimination between Patients with and without Tumors}

In our study, with a cut-off fixed in $37 \mathrm{IU} / \mathrm{mL}, \mathrm{CA} 19-9$ showed a good diagnostic capacity to differentiate patients with tumors (dCCA+PDAC) from healthy individuals, with an AUC of 0.93 in both cohorts. However, as shown in Figure 5A, it was not so good in differentiating between $\mathrm{dCCA}+\mathrm{PDAC}$ and patients without cancer (Control+BPD). AUC was 0.845, 0.820 and 0.828 in discovery, validation and the whole cohort, respectively (Figure 5B). 

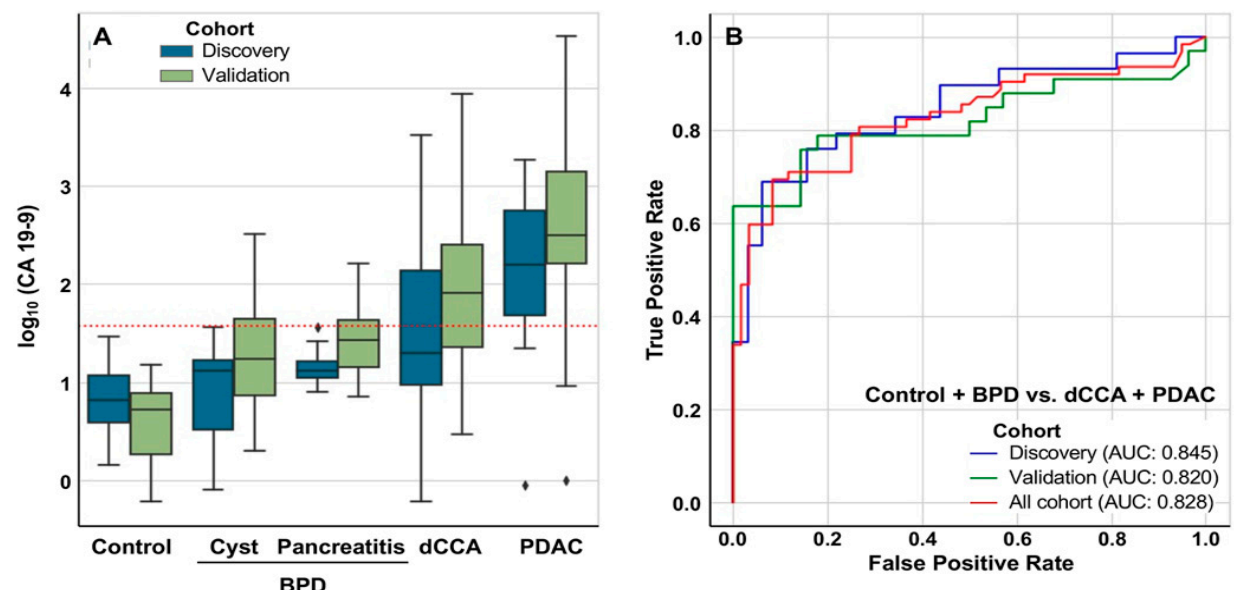

Figure 5. Diagnostic prediction capacity of CA 19-9 in tumors (dCCA+PDAC) vs. non tumors (Control+BPD). (A) Box plot diagrams show the $\log _{10}$ CA 19-9 (cut-off of $37 \mathrm{IU} / \mathrm{mL}$ ). (B) Area under the receiver operating characteristic curve (AUC) in discovery and validation cohorts and considering all cohorts.

As shown in Figure 6, a model including 10 metabolites [amino acids sarcosine, tryptophan and aspartic acid, lysophosphatidylethanolamine $\mathrm{PE}(0: 0 / 16: 0)$, phosphatidylinositol PI(18:0/18:2), diglycerides DG(38:4) and DG(34:0), sphingomyelin SM(42:1), N-acyl ethanolamine NA(16:0) and sterol pregnenolone sulfate] was generated to differentiate patients with tumors (dCCA+PDAC) and without malignancies (Control+BPD).
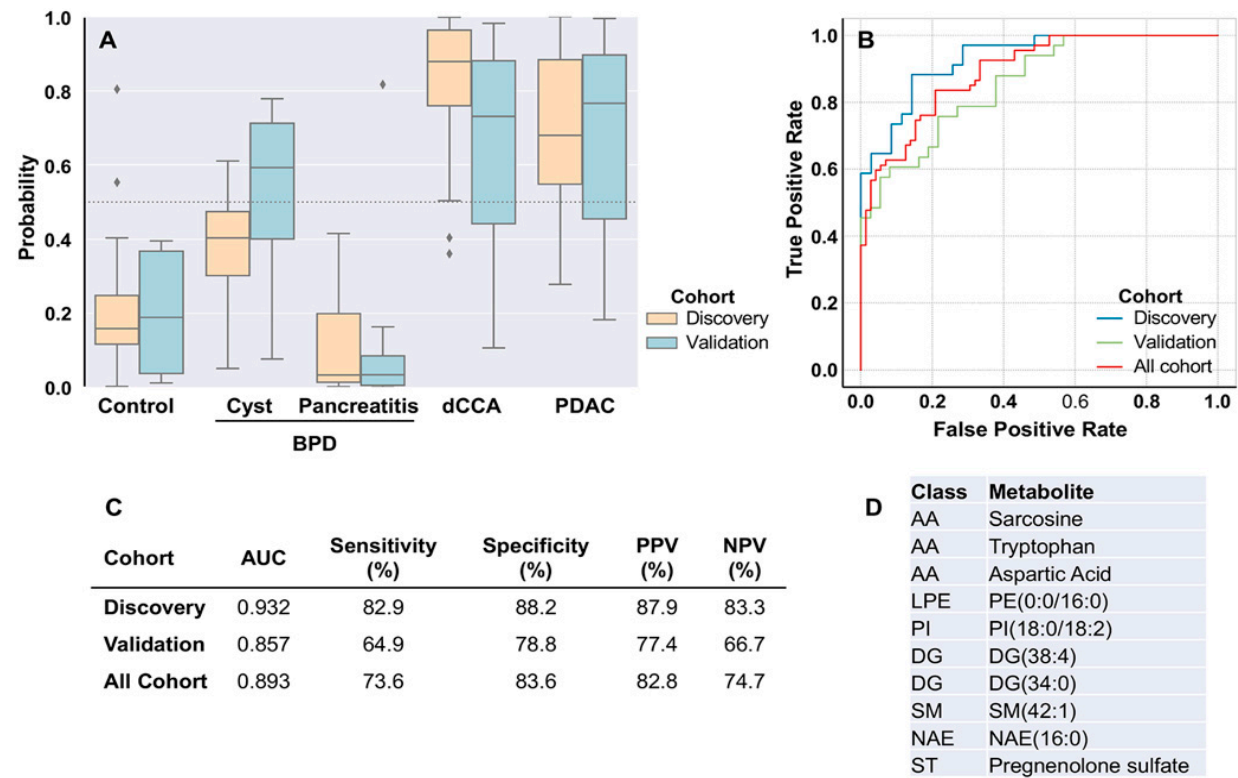

Figure 6. Diagnostic prediction capacity of the logistic model in tumors (dCCA+PDAC) vs. non tumors (Control+BPD). (A) Box plot diagrams showing the probability to detect each group as tumors. (B) Area under the receiver operating characteristic curve (AUC) in discovery and validation cohorts and considering all cohorts. (C) AUC, sensitivity, specificity, positive predictive value (PPV) and negative predictive value (NPV) of the algorithm to differentiate tumors vs. non tumors in each cohort. (D) Selected metabolites included in the model. AA, amino acids; DG, diglycerides; LPE, lysophosphatidylethanolamines; NAE, N-acyl ethanolamines; PI, phosphatidylinositols; SM, sphingomyelins; ST, steroids.

Using this model, the probability of diagnosing patients with chronic pancreatitis or healthy subjects as individuals suffering from dCCA or PDAC is low. However, this risk is higher for patients 
with benign pancreatic cysts (Figure 6A). AUC was 0.93 in discovery, 0.86 in validation and 0.89 considering the whole cohort. Sensitivity was $73.6 \%$ and specificity $83.6 \%$ considering the whole cohort. We have evaluated the relationship between the age and the diagnostic error rate of the model. Based on a stratification of the patients in quantiles, the diagnostic error rate was constant and around $20 \%$ (average $21 \%$, ranging from the $17 \%$ to $29 \%$ ) and was not associated with the patient's age.

In our study, CA $19-9$ showed a sensitivity of $71 \%$ and a specificity of $83 \%$ to differentiate patients with tumors from individuals without tumors (Controls+BPD).

\subsection{Discrimination between $\mathrm{dCC} A$ and PDAC}

Since none of the individual circulating metabolites had a sufficient capability of distinguishing dCCA from PDAC (Table 3), our next goal was to obtain a predictive model for discriminating between both tumors. A logistic regression model was built with nine metabolites (Figure S1) (acylcarnitine AC(16:0), ceramide Cer(d18:1/24:0), phosphatidylcholines PC(20:0/0:0) and PC(O-16:0/20.3), lysophosphatidylcholines PC(20:0/0:0) and PC(0:0/20:0), lysophosphatidylethanolamine PE(P-18:2/0:0), and sphingomyelins $\mathrm{SM}(\mathrm{d} 18: 2 / 22: 0)$ and $\mathrm{SM}(\mathrm{d} 18: 2 / 23: 0))$, with an AUC of 0.91 in discovery, 0.82 in validation and 0.85 considering the whole cohort; sensitivity was $55.9 \%$ and specificity $89.5 \%$ considering all the patients. The analysis of CA 19-9 showed a sensitivity of $77 \%$ and a specificity of $48 \%$ to differentiate patients with PDAC from those with dCCA (Figure S2).

Another logistic regression model was built with the nine metabolites plus CA 19-9 (Figure 7), which improved the sensitivity. However, the specificity slightly decreased in the full cohort and especially in the validation cohort. Thus, AUC was 0.888 , sensitivity $71.4 \%$ and specificity 89.2 considering the whole cohort.
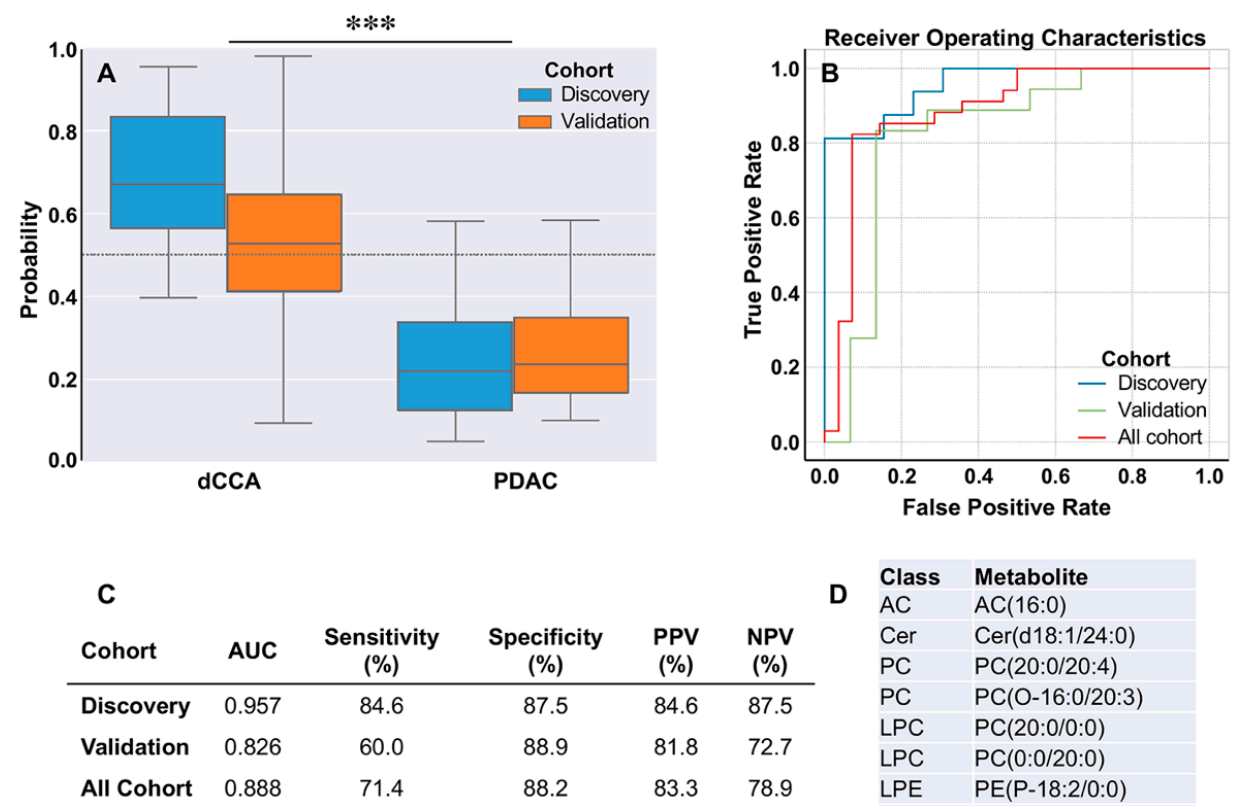

$\begin{array}{ll}\text { Class } & \text { Metabolite } \\ \text { AC } & \text { AC(16:0) } \\ \text { Cer } & \text { Cer(d18:1/24:0) } \\ \text { PC } & \text { PC(20:0/20:4) } \\ \text { PC } & \text { PC(O-16:0/20:3) } \\ \text { LPC } & \text { PC }(20: 0 / 0: 0) \\ \text { LPC } & \text { PC }(0: 0 / 20: 0) \\ \text { LPE } & \text { PE(P-18:2/0:0) } \\ \text { SM } & \text { SM(d18:2/22:0) } \\ \text { SM } & \text { SM(d18:2/23:0) } \\ \text { +CA 19-9 }\end{array}$

Figure 7. Diagnostic prediction capacity of the logistic model in dCCA vs. PDAC. (A) Box plot diagrams showing the probability to detect each type of tumor. (B) Area under the receiver operating characteristic curve (AUC) in discovery and validation cohorts and considering the whole cohort. (C) AUC, sensitivity, specificity, positive predictive value (PPV) and negative predictive value (NPV) of the algorithm to differentiate dCCA vs. PDAC in each cohort. (D) Selected metabolites included in the model: AC, acylcarnitine; Cer, ceramide; LPC, lysophosphatidylcholines; LPE, lysophosphatidylethanolamines; PC, phosphatidylcholines; SM, sphingomyelins plus CA 19-9. ${ }^{* *}, p<0.001$. 


\section{Discussion}

The lack of non-invasive biomarkers for the early diagnosis of PDAC and dCCA contributes to the bad prognosis of these tumors [12]. The anatomical difficulty in accessing the tumors to obtain good quality biopsies for diagnostic purposes makes it necessary to identify minimally invasive biomarkers that could help, not only in the early detection of these tumors to enable more patients to benefit from surgical treatment, but also in the prognosis and follow-up of these patients during treatment. However, although important efforts have been made during recent years, none of the identified markers have been validated and reached clinical practice. Despite their moderate clinical utility, only CA 19-9 and carcinoembryonic antigen (CEA) are currently used for PDAC and CCA diagnosis [13].

Omics technologies are providing valuable information to understand cancer biology. Metabolic reprogramming is one hallmark of tumor cells $[14,15]$; thus, the analysis of the metabolome (hundreds of small molecules or metabolites) in body fluids of patients with cancer can give an indirect reflection of the metabolic behavior of the tumors and could be used to identify potential biomarkers.

Several studies have been conducted to identify serum metabolomic profiles for the diagnosis of pancreatic or biliary cancers. Most of them included only patients with pancreatic cancer and healthy controls [16-18] or with biliary cancer and healthy individuals [19]. However, it is important to include clinically relevant controls since the metabolome can be affected by many factors, including gender, age, comorbidities, medication, life style, environment or circadian rhythms; in fact, important intra-day variations have been observed in serum levels of patients with advanced pancreatic cancer, which were further affected by cachexia [20].

The use of metabolomics to discriminate between different types of tumors and between tumors and benign diseases has been less explored. Combinations of metabolites discriminating malignant from benign pancreaticobiliary diseases and from healthy controls have been reported, although the number of cases was low and most of the patients with tumors were in an advanced stage, for which their usefulness in early diagnosis cannot be guaranteed [21]. More recently, a biomarker signature for the differential diagnosis between PDAC and chronic pancreatitis was reported, consisting of nine metabolites, five of them lipids (two sphingomyelins, sphinganine 1-phosphate, one phosphatidylcholine and one ceramide), and proline, histidine, pyruvate and isocitrate plus CA 19-9, with a negative predictive value of $99.9 \%$ in patients with chronic pancreatitis [22].

All these studies support the concept that the combination of several metabolite markers allows for a more accurate diagnosis. In this study, we have included patients with biopsy-proven tumors or cysts located in the head of the pancreas divided into two independent cohorts of PDAC, dCCA, BPD and controls. Although serum bile acids levels represented the most marked alteration in patients with cancer, this hypercholanemic condition occurs in different pathologies that are accompanied by cholestasis, in which compensatory mechanisms are developed to limit the accumulation and toxic effects of these compounds [23]. It has been demonstrated that obstructive jaundice impacts the performance of biomarkers for PDAC [24], and in our study, a certain degree of cholestasis was found in some patients with tumors, since serum bilirubin was elevated, and as a consequence, none of the bile acid species measured could be considered as a good biomarker.

In the present study we have identified a multimarker signature for the differential diagnosis of adenocarcinomas located in the pancreas including nine metabolites plus CA 19-9 with better performance than serum CA 19-9 alone and another panel of ten metabolites (seven lipids and three amino acids) with similar performance to serum CA 19-9 to discriminate tumors from BPD but which are especially useful for chronic pancreatitis. Since this disease is a risk factor for the development of pancreatic cancer [25], these biomarkers could be useful for early detection of tumor development, for monitoring patients during treatment and for avoiding unnecessary pancreatic surgery and its complications. Interestingly, some of the metabolites included in the signature proposed here belonged to the same families of compounds (amino acids, sphingomyelins and ceramides) of a previously described model [22]. Changes in serum levels of certain amino acids have been described in other tumors, such as liver [26,27] and breast [28] cancer. In addition, sphingomyelins and ceramides have 
been found altered in the serum of patients with liver [27] and ovarian [29] cancer. Alterations in sphingolipid metabolism have been associated with cell proliferation [30]. Our model of changes in ten metabolites was less accurate than CA 19-9 levels in distinguishing pancreatic cysts from tumors in the head of pancreas, although the low number of cases of cystic lesions in our cohort can be considered a limitation. In recent years, several studies have proposed circulating microRNA (miRNA) signatures for early detection of pancreatic cancer [31] or for the differential diagnosis of PDAC and chronic pancreatitis with good sensitivity and specificity [32], although none of them included a group of patients with pancreatic cysts. A recent study proposed a two-miRNA panel of downregulated miR-16 and upregulated miR-877 to differentiate patients with dCCA from benign disease (AUC $=0.90$ ) and from PDAC $(A U C=0.88)$ [33]. Serum proteins have also been investigated. The analysis of cell migration-inducing hyaluronan binding protein (CEMIP) plus CA 19-9 improved the diagnostic value compared to CA 19-9 alone for the diagnosis of pancreatic cancer [34]; the study included a small but very heterogeneous group of patients with BPD in the control cohort, but the results must be validated.

In sum, in this study, using two independent cohorts of patients, we have identified a model consisting of 9 metabolites in serum with promising capability to differentiate both types of pancreatic head adenocarcinomas, with AUC $=0.854$. Because accurate diagnosis of these tumors remains challenging, our results suggest that the analysis of multiple types of biomarkers could help in the early and differential diagnosis and in the follow-up of these aggressive tumors.

\section{Materials and Methods}

\subsection{Study Population and Eligibility}

Fasting serum samples from dCCA $(n=34), \operatorname{PDAC}(n=38)$, BPD $(n=42)$, and healthy subjects $(n$ $=25$ ) were obtained from two Spanish hospitals; University Hospital of Salamanca, National DNA Bank Carlos III, and Donostia University Hospital in San Sebastian. Samples were randomly divided in two cohorts, "discovery" and "validation", with equal proportional representation of individuals belonging to each pathology as well as to each origin of samples.

Inclusion criteria for patients with dCCA and PDAC were histopathologic confirmation of diagnosis by expert pathologists and serum obtained before any type of treatment. Exclusion criteria were other types of CCA or synchronous presence of another type of malignancy. The BPD group included 22 samples from patients with cysts and 20 from patients with chronic pancreatitis. Selected healthy individuals had no history of any type of malignancy and no clinical evidence of hepatopancreaticobiliary disease. Clinical and laboratory test values were collected from the patients' records. The research protocol was approved by the Ethics Committee for Clinical Research of Salamanca (July 18, 2018) and San Sebastian (October 16, 2019), and informed written consent for the samples to be used for biomedical research was obtained from each patient.

\subsection{Metabolomic Analyses}

Serum metabolic profiles were analyzed as previously described [35]. Briefly, two ultrahigh-performance liquid chromatography (UHPLC)-time of flight-MS based platforms analyzing methanol and chloroform/methanol serum extracts were combined with the amino acid measurement using an UHPLC-single quadrupole-MS based analysis. Identified ion features in the methanol extract platform included amino acids and its derivatives and lipids.

Metabolite extraction procedures, chromatographic separation conditions and mass spectrometric detection conditions have been previously described [35]. Metabolomics data were pre-processed using the TargetLynx application manager for MassLynx 4.1 (Waters Corp., Milford, MA, USA). Intraand inter-batch normalization was performed by inclusion of multiple internal standards and pool calibration response correction, following a previously described procedure [36]. Data quality was assessed by the inclusion of quality control samples, including repeated injections of these samples to evaluate the reproducibility of the analysis process [36]. 


\subsection{Statistical Analysis}

Data are shown as mean \pm SD. Differences between groups were determined using the Student's $t$-test or the Bonferroni method of multiple range test, as appropriate. Calculations were performed using the statistical software package R v.3.4.0 (R Development Core Team, 2017; http://cran.r-project.org).

Multivariate principal component analysis (PCA) [37] and orthogonal partial least squares discriminant analysis (OPLS-DA) [38] modeling were performed with the software SIMCA 14.1 (Umetrics, Malmo, Sweden). Model quality was assessed using $\mathrm{R}^{2}$ and $\mathrm{Q}^{2}$ values, which indicate the explained fraction of variance and the goodness of prediction, respectively. The $\mathrm{Q}^{2}$ parameter was calculated by sevenfold cross validation.

To find statistical models to differentiate patients with tumors (dCCA or PDAC) and subjects without tumors (controls or BPD [chronic pancreatitis or pancreatic cysts]), as well as to differentiate each type of tumor, dCCA vs. PDAC, generalized linear models (GLM) were used and those selected were confirmed by leave-one-out cross validation (LOOCV). Box-Cox transformations were applied to the biomarker metabolite levels for correcting non-normally distributed data and used to calculate the classification algorithm. The diagnostic accuracy of the model to identify patients in each comparison was assessed using the AUC $p<0.05$.

\section{Conclusions}

Based on the results obtained in the present study, we propose novel specific panels of serum metabolites that can help in the early and differential diagnosis of dCCA and PDAC. Further validation of their clinical usefulness in prospective studies including other relevant controls and in combination with clinical features is required.

Supplementary Materials: The following are available online at http://www.mdpi.com/2072-6694/12/6/1433/s1, Figure S1: Diagnostic prediction capacity of the model of nine metabolites in dCCA vs. PDAC, Figure S2: Diagnostic prediction capacity of CA 19-9 in dCCA vs. PDAC, Table S1: Diagnostic capacity of the metabolites in the comparison.

Author Contributions: Conceptualization, R.I.R.M., J.J.G.M., J.M.B., L.M.-B. and L.B.; methodology, R.I.R.M., J.J.G.M., A.S.-M., E.A., I.M.-A., C.A.; software, E.A., I.M.-A., C.A.; data curation, R.I.R.M., L.M.-B., A.L., A.L.C., L.M.G., M.L.G.; writing—original draft preparation, R.I.R.M. and J.J.G.M.; writing-review and editing, all authors; visualization, all authors; project administration, R.I.R.M.; funding acquisition, R.I.R.M., J.J.G.M., J.M.B., M.A.A., M.L.M.-C. All authors have read and agreed to the published version of the manuscript.

Funding: This study was supported by the Centro Internacional sobre el Envejecimiento, Spain (OLD-HEPAMARKER, 0348_CIE_6_E) co-financed with European Union ERDF funds; Carlos III Institute of Health, Spain (PI16/00598, PI16/01126, PI18/01075, PI19/00819) and Miguel Servet Program (CON14/00129) co-financed by European Regional Development Fund; Asociación Española Contra el Cancer, Spain (AECC-Cánceres raros 2017/2020); H2020 ESCALON project: H2020-SC1-BHC-2018-2020; Fundacion La Caixa (Hepacare Project); MCIU/AEI/FEDER, EU (SAF2017-87301-R); Severo Ochoa Excellence Accreditation (SEV-2016-0644). A. Sanchez-Martin and A. Lapitz were supported by pre-doctoral scholarships funded by the Ministry of Science, Innovation and Universities (FPU17/04027) and the Basque Government (PRE_2017_1_0345), respectively, and M.L. Gutiérrez is supported by the "Stop fuga de Cerebros" grant from ROCHE FARMA SA. This work was carried out in the framework of Working Group 5 of the COST Action CA18122, European Cholangiocarcinoma Network, EURO-CHOLANGIO-NET.

Conflicts of Interest: Enara Arretxe and Cristina Alonso and Ibon Martínez-Arranz are employed by OWL Metabolomics and Jesus M. Banales is a member of the scientific advisory board of OWL Metabolomics. 


\section{Abbreviations}

$\begin{array}{ll}\text { AUC } & \begin{array}{l}\text { area under the receiver operating characteristic curve } \\ \text { benign pancreatic disease } \\ \text { BPD }\end{array} \\ \text { CA 19-9 } & \begin{array}{l}\text { cholanydrate antigen 19-9 } \\ \text { distal cholangiocarcinoma } \\ \text { CCA }\end{array} \\ \text { dCCA } & \text { orthogonal partial least squares } \\ \text { OPLS } & \text { principal component analysis } \\ \text { PCA } & \text { pancreatic ductal adenocarcinoma } \\ \text { PDAC } & \text { ultra-high performance liquid chromatography coupled to mass spectrometry }\end{array}$

\section{References}

1. Ethun, C.G.; Lopez-Aguiar, A.G.; Pawlik, T.M.; Poultsides, G.; Idrees, K.; Fields, R.C.; Weber, S.M.; Cho, C.; Martin, R.C.; Scoggins, C.R.; et al. Distal Cholangiocarcinoma and Pancreas Adenocarcinoma: Are They Really the Same Disease? A 13-Institution Study from the US Extrahepatic Biliary Malignancy Consortium and the Central Pancreas Consortium. J. Am. Coll. Surg. 2017, 224, 406-413. [CrossRef] [PubMed]

2. Siegel, R.L.; Miller, K.D.; Jemal, A. Cancer statistics, 2015. CA Cancer J. Clin. 2015, 65, 5-29. [CrossRef] [PubMed]

3. Siegel, R.L.; Miller, K.D.; Jemal, A. Cancer statistics, 2019. CA Cancer J. Clin. 2019, 69, 7-34. [CrossRef] [PubMed]

4. Banales, J.M.; Cardinale, V.; Carpino, G.; Marzioni, M.; Andersen, J.B.; Invernizzi, P.; Lind, G.E.; Folseraas, T.; Forbes, S.J.; Fouassier, L.; et al. Expert consensus document: Cholangiocarcinoma: Current knowledge and future perspectives consensus statement from the European Network for the Study of Cholangiocarcinoma (ENS-CCA). Nat. Rev. Gastroenterol. Hepatol. 2016, 13, 261-280. [CrossRef]

5. Marin, J.J.G.; Lozano, E.; Herraez, E.; Asensio, M.; Di Giacomo, S.; Romero, M.R.; Briz, O.; Serrano, M.A.; Efferth, T.; Macias, R.I.R. Chemoresistance and chemosensitization in cholangiocarcinoma. Biochim Biophys Acta Mol. Basis Dis. 2018, 1864, 1444-1453. [CrossRef]

6. Forner, A.; Vidili, G.; Rengo, M.; Bujanda, L.; Ponz-Sarvise, M.; Lamarca, A. Clinical presentation, diagnosis and staging of cholangiocarcinoma. Liver Int. 2019, 39 (Suppl. 1), 98-107. [CrossRef]

7. Fong, Z.V.; Winter, J.M. Biomarkers in pancreatic cancer: Diagnostic, prognostic, and predictive. Cancer J. 2012, 18, 530-538. [CrossRef]

8. Macias, R.I.R.; Banales, J.M.; Sangro, B.; Muntane, J.; Avila, M.A.; Lozano, E.; Perugorria, M.J.; Padillo, F.J.; Bujanda, L.; Marin, J.J.G. The search for novel diagnostic and prognostic biomarkers in cholangiocarcinoma. Biochim. Biophys. Acta Mol. Basis Dis. 2018, 1864, 1468-1477. [CrossRef]

9. Macias, R.I.R.; Kornek, M.; Rodrigues, P.M.; Paiva, N.A.; Castro, R.E.; Urban, S.; Pereira, S.P.; Cadamuro, M.; Rupp, C.; Loosen, S.H.; et al. Diagnostic and prognostic biomarkers in cholangiocarcinoma. Liver Int. 2019, 39 (Suppl. 1), 108-122. [CrossRef]

10. Boone, B.A.; Steve, J.; Zenati, M.S.; Hogg, M.E.; Singhi, A.D.; Bartlett, D.L.; Zureikat, A.H.; Bahary, N.; Zeh, H.J., 3rd. Serum CA 19-9 response to neoadjuvant therapy is associated with outcome in pancreatic adenocarcinoma. Ann. Surg. Oncol. 2014, 21, 4351-4358. [CrossRef]

11. Tempero, M.A.; Uchida, E.; Takasaki, H.; Burnett, D.A.; Steplewski, Z.; Pour, P.M. Relationship of carbohydrate antigen 19-9 and Lewis antigens in pancreatic cancer. Cancer Res. 1987, 47, 5501-5503. [PubMed]

12. Frampton, A.E.; Giovannetti, E. Diagnosing pancreatic ductal adenocarcinoma using plasma extracellular vesicle RNA profiles. Gut 2020, 69, 404-405. [CrossRef] [PubMed]

13. Liang, B.; Zhong, L.; He, Q.; Wang, S.; Pan, Z.; Wang, T.; Zhao, Y. Diagnostic Accuracy of Serum CA19-9 in Patients with Cholangiocarcinoma: A Systematic Review and Meta-Analysis. Med. Sci. Monit. 2015, 21, 3555-3563. [CrossRef] [PubMed]

14. Cairns, R.A.; Harris, I.S.; Mak, T.W. Regulation of cancer cell metabolism. Nat. Rev. Cancer 2011, 11, 85-95. [CrossRef]

15. Satriano, L.; Lewinska, M.; Rodrigues, P.M.; Banales, J.M.; Andersen, J.B. Metabolic rearrangements in primary liver cancers: Cause and consequences. Nat. Rev. Gastroenterol. Hepatol. 2019, 16, 748-766. [CrossRef] 
16. OuYang, D.; Xu, J.; Huang, H.; Chen, Z. Metabolomic profiling of serum from human pancreatic cancer patients using $1 \mathrm{H}$ NMR spectroscopy and principal component analysis. Appl. Biochem. Biotechnol. 2011, 165, 148-154. [CrossRef]

17. Akita, H.; Ritchie, S.A.; Takemasa, I.; Eguchi, H.; Pastural, E.; Jin, W.; Yamazaki, Y.; Goodenowe, D.B.; Nagano, H.; Monden, M.; et al. Serum Metabolite Profiling for the Detection of Pancreatic Cancer: Results of a Large Independent Validation Study. Pancreas 2016, 45, 1418-1423. [CrossRef]

18. Di Gangi, I.M.; Mazza, T.; Fontana, A.; Copetti, M.; Fusilli, C.; Ippolito, A.; Mattivi, F.; Latiano, A.; Andriulli, A.; Vrhovsek, U.; et al. Metabolomic profile in pancreatic cancer patients: A consensus-based approach to identify highly discriminating metabolites. Oncotarget 2016, 7, 5815-5829. [CrossRef]

19. Liang, Q.; Liu, H.; Zhang, T.; Jiang, Y.; Xing, H.; Zhang, H. Serum metabolomics uncovering specific metabolite signatures of intra- and extrahepatic cholangiocarcinoma. Mol. Biosyst. 2016, 12, 334-340. [CrossRef]

20. Fujiwara, Y.; Kobayashi, T.; Chayahara, N.; Imamura, Y.; Toyoda, M.; Kiyota, N.; Mukohara, T.; Nishiumi, S.; Azuma, T.; Yoshida, M.; et al. Metabolomics evaluation of serum markers for cachexia and their intra-day variation in patients with advanced pancreatic cancer. PLoS ONE 2014, 9, e113259. [CrossRef]

21. Itoi, T.; Sugimoto, M.; Umeda, J.; Sofuni, A.; Tsuchiya, T.; Tsuji, S.; Tanaka, R.; Tonozuka, R.; Honjo, M.; Moriyasu, F; et al. Serum Metabolomic Profiles for Human Pancreatic Cancer Discrimination. Int. J. Mol. Sci. 2017, 18, 767. [CrossRef] [PubMed]

22. Mayerle, J.; Kalthoff, H.; Reszka, R.; Kamlage, B.; Peter, E.; Schniewind, B.; Gonzalez Maldonado, S.; Pilarsky, C.; Heidecke, C.D.; Schatz, P.; et al. Metabolic biomarker signature to differentiate pancreatic ductal adenocarcinoma from chronic pancreatitis. Gut 2018, 67, 128-137. [CrossRef] [PubMed]

23. Marin, J.J.; Macias, R.I.; Briz, O.; Banales, J.M.; Monte, M.J. Bile Acids in Physiology, Pathology and Pharmacology. Curr. Drug Metab. 2015, 17, 4-29. [CrossRef] [PubMed]

24. Tonack, S.; Jenkinson, C.; Cox, T.; Elliott, V.; Jenkins, R.E.; Kitteringham, N.R.; Greenhalf, W.; Shaw, V.; Michalski, C.W.; Friess, H.; et al. iTRAQ reveals candidate pancreatic cancer serum biomarkers: Influence of obstructive jaundice on their performance. Br. J. Cancer 2013, 108, 1846-1853. [CrossRef]

25. Lowenfels, A.B.; Maisonneuve, P.; Cavallini, G.; Ammann, R.W.; Lankisch, P.G.; Andersen, J.R.; Dimagno, E.P.; Andren-Sandberg, A.; Domellof, L. Pancreatitis and the risk of pancreatic cancer. International Pancreatitis Study Group. N. Engl. J. Med. 1993, 328, 1433-1437. [CrossRef]

26. Gao, R.; Cheng, J.; Fan, C.; Shi, X.; Cao, Y.; Sun, B.; Ding, H.; Hu, C.; Dong, F.; Yan, X. Serum Metabolomics to Identify the Liver Disease-Specific Biomarkers for the Progression of Hepatitis to Hepatocellular Carcinoma. Sci. Rep. 2015, 5, 18175. [CrossRef]

27. Banales, J.M.; Inarrairaegui, M.; Arbelaiz, A.; Milkiewicz, P.; Muntane, J.; Munoz-Bellvis, L.; La Casta, A.; Gonzalez, L.M.; Arretxe, E.; Alonso, C.; et al. Serum Metabolites as Diagnostic Biomarkers for Cholangiocarcinoma, Hepatocellular Carcinoma, and Primary Sclerosing Cholangitis. Hepatology 2019, 70, 547-562. [CrossRef]

28. Eniu, D.T.; Romanciuc, F.; Moraru, C.; Goidescu, I.; Eniu, D.; Staicu, A.; Rachieriu, C.; Buiga, R.; Socaciu, C. The decrease of some serum free amino acids can predict breast cancer diagnosis and progression. Scand. J. Clin. Lab. Investig. 2019, 79, 17-24. [CrossRef]

29. Kozar, N.; Kruusmaa, K.; Bitenc, M.; Argamasilla, R.; Adsuar, A.; Goswami, N.; Arko, D.; Takac, I. Metabolomic profiling suggests long chain ceramides and sphingomyelins as a possible diagnostic biomarker of epithelial ovarian cancer. Clin. Chim. Acta 2018, 481, 108-114. [CrossRef]

30. Morad, S.A.; Cabot, M.C. Ceramide-orchestrated signalling in cancer cells. Nat. Rev. Cancer 2013, 13, 51-65. [CrossRef]

31. Vila-Navarro, E.; Duran-Sanchon, S.; Vila-Casadesus, M.; Moreira, L.; Gines, A.; Cuatrecasas, M.; Lozano, J.J.; Bujanda, L.; Castells, A.; Gironella, M. Novel Circulating miRNA Signatures for Early Detection of Pancreatic Neoplasia. Clin. Transl. Gastroenterol. 2019, 10, e00029. [CrossRef] [PubMed]

32. Schultz, N.A.; Dehlendorff, C.; Jensen, B.V.; Bjerregaard, J.K.; Nielsen, K.R.; Bojesen, S.E.; Calatayud, D.; Nielsen, S.E.; Yilmaz, M.; Hollander, N.H.; et al. MicroRNA biomarkers in whole blood for detection of pancreatic cancer. JAMA 2014, 311, 392-404. [CrossRef] [PubMed]

33. Meijer, L.L.; Puik, J.R.; Le Large, T.Y.S.; Heger, M.; Dijk, F.; Funel, N.; Wurdinger, T.; Garajova, I.; van Grieken, N.C.T.; van de Wiel, M.A.; et al. Unravelling the Diagnostic Dilemma: A MicroRNA Panel of 
Circulating MiR-16 and MiR-877 as A Diagnostic Classifier for Distal Bile Duct Tumors. Cancers 2019, 11, 1181. [CrossRef] [PubMed]

34. Lee, H.S.; Jang, C.Y.; Kim, S.A.; Park, S.B.; Jung, D.E.; Kim, B.O.; Kim, H.Y.; Chung, M.J.; Park, J.Y.; Bang, S.; et al. Combined use of CEMIP and CA 19-9 enhances diagnostic accuracy for pancreatic cancer. Sci. Rep. 2018, 8, 3383. [CrossRef] [PubMed]

35. Barr, J.; Caballeria, J.; Martinez-Arranz, I.; Dominguez-Diez, A.; Alonso, C.; Muntane, J.; Perez-Cormenzana, M.; Garcia-Monzon, C.; Mayo, R.; Martin-Duce, A.; et al. Obesity-dependent metabolic signatures associated with nonalcoholic fatty liver disease progression. J. Proteome Res. 2012, 11, 2521-2532. [CrossRef] [PubMed]

36. Martinez-Arranz, I.; Mayo, R.; Perez-Cormenzana, M.; Minchole, I.; Salazar, L.; Alonso, C.; Mato, J.M. Enhancing metabolomics research through data mining. J. Proteom. 2015, 127, 275-288. [CrossRef]

37. Jolliffe, I.T. Principal Component Analysis; Springer: New York, NY, USA, 2002.

38. Trygg, J.; Wold, S. Orthogonal projections to latent structures (O-PLS). J. Chemom. 2002, 16, 119-128. [CrossRef]

(C) 2020 by the authors. Licensee MDPI, Basel, Switzerland. This article is an open access article distributed under the terms and conditions of the Creative Commons Attribution (CC BY) license (http://creativecommons.org/licenses/by/4.0/). 